\title{
Hominin lower second premolar morphology: evolutionary inferences through geometric morphometric analysis
}

\author{
M. Martinón-Torres ${ }^{\text {a,* }}$, M. Bastir ${ }^{\text {b,c }}$, J.M. Bermúdez de Castro a , A. Gómez ${ }^{\text {a }}$, \\ S. Sarmiento ${ }^{\text {d }}$, A. Muela ${ }^{\text {d, J.L. Arsuaga }}{ }^{\mathrm{e}}$ \\ b $\quad$ a Centro Nacional de Investigación sobre Evolución Humana (CENIEH), C/Toledo 4-5²,09004 Burgos, Spain \\ Department of Palaeobiology, Museo Nacional de Ciencias Naturales, CSIC. C/José Gutiérrez Abascal 2, 28006 Madrid, Spain \\ e Hull York Medical School, The University of York, Heslington, York YO10 5DD, United Kingdom \\ ${ }^{\mathrm{d}}$ Fundación Atapuerca. C/Condestable 2, $4{ }^{\circ}$ C, 09004 Burgos, Spain \\ Centro de Evolución y Comportamiento Humanos. C/Sinesio Delgado 4, pabellón 14, 28029 Madrid, Spain
}

\begin{abstract}
Mandibular premolars are increasingly used in taxon-specific diagnostic analyses of hominins. Among the principal difficulties in these evaluations is the absence of discrete, discernible, and comparable anatomical structures for rigorous quantitative assessment. Previous research has addressed either internal crown surface features (such as cusps and fossae) or the morphology of the crown outline. In the present paper, we integrate both types of information in the examination of morphological variation of lower P4s ( $\mathrm{n} 1 / 496$ ) among various fossil hominin species with an emphasis on genus Homo. We use a set of 34 2D landmarks combining coordinate data from four classical dental landmarks on the occlusal surface and 30 sliding semilandmarks of the crown outline. Our results indicate that external shape variation is closely related to the configuration of the occlusal morphological features and influenced by dental size. The external and internal shapes of P4 are polymorphic but still useful in depicting a primitive-derived gradient. The primitive pattern seems to have been an asymmetrical contour with a mesially displaced metaconid, development of a bulging talonid, and a broad occlusal polygon. The trend toward dental reduction during the Pleistocene produced different morphological variants with a reduced occlusal polygon and decreased lingual occlusal surface in later Homo species. Homo heidelbergensis/neanderthalensis have fixed plesiomorphic traits in high percentages, whereas in modern humans a symmetrical outline with a centered metaconid and talonid reduction evolved.
\end{abstract}

Keywords: Procrustes; Sliding semilandmarks; Occlusal shape; Premolars; Asymmetry

Introduction

The morphology of mandibular premolars may be taxonomically diagnostic among hominin taxa (Ludwig, 1957; Patte, 1962; Biggerstaff, 1969; Wood and Uytterschaut, 1987; Uytterschaut and Wood, 1989; Bailey, 2002a,b; Bailey and Lynch, 2005). However, capturing premolar occlusal morphology in a way that allows rigorous quantitative comparison remains a challenge. Research on premolar crown morphology has largely relied on descriptive and qualitative assessments of the shape of the crown and the cuspal morphology (e.g., Fraipont and Lohest, 1887; Genet-Varcin, 1962; Lumley et al., 1972; Turner et al., 1991; Irish, 1993; Scott and Turner, 1997). Recently, Elliptic Fourier Analysis (EFA) (Lestrel, 1974, 1997; Khul and Giardina, 1982; Seiffert and Kappelman, 2001) has been used to quantify the crown outline (Bailey and Lynch, 2005). While this method is useful in obtaining mean shapes for inter-hominin comparisons (Bailey and Lynch, 2005), it does not provide geometrically comparable structures for a direct comparison. In addition, EFA provides no informa-tion about size.

These analytical problems can be overcome to some degree by using recent improvements in geometric morphometric 
techniques including sliding semilandmarks (Bookstein, 1997). Semilandmarks are evenly spaced points along an out-line that can be analyzed together with information about cusp morphology by common Procrustes superimposition tech-niques (Rohlf and Slice, 1990; Bookstein, 1991). Thus, these methods allow investigation on both shape and size (see details in Materials and methods).

The morphology of the lower second premolar (P4) has been described as particularly useful for taxonomic designations in hominins (e.g., Wood and Uytterschaut, 1987; Bailey and Lynch, 2005). For this reason, we use geometric morpho-metric techniques to explore the utility of P4 morphology in characterizing Homo species. We consider dental variation in Middle and Late Pleistocene groups and include small samples of Australopithecus, Pliocene, and Lower Pleistocene Homo for comparison.

\section{Materials and methods}

We used standardized occlusal surface pictures of $96 \mathrm{P} 4 \mathrm{~s}$ sampled from various hominin taxa (Table 1). We focus on premolar variation in Middle and Late Pleistocene Homo. In order to assess the phylogenetic significance of these differences, data from small samples of Australopithecus and Pliocene and Lower Pleistocene Homo are also included. In order to investigate trait polarity, we have assumed that Australopithecus represents the primitive condition. For comparative purposes, we have pooled some specimens into more or less homogeneous groups relating their geographical

Table 1

List of the specimens included in this analysis

Australopithecus $(\mathrm{n}=6)$
Paranthropus $(\mathrm{n}=1)$
Plio-Pleistocene
Homo $(\mathrm{n}=6)$
Late Lower Pleistocene
Homo $(\mathrm{n}=3)$
African Middle
Pleistocene Homo $(\mathrm{n}=5)$
Homo antecessor $(\mathrm{n}=2)$
European Middle Pleistocene
Homo $(\mathrm{n}=23)$

AL-266, AL-333w, AL-400,

Sts14, Sts52, Stw 498 (casts)

TM1517 (cast)

$\mathrm{OH}-7, \mathrm{OH}-13, \mathrm{OH}-16$ (casts)

D211, D2735 (originals)

KNM-ER 1802 (cast)

KNM-WT 15000, KNM-ER

992, Sangiran 6 (casts)

Ternifine 1, 2, 3 (originals)

Rabat, OH-22 (casts)

ATD6-4, ATD6-96 (originals)

Mauer (cast)

Arago 13, Arago 28 (originals)

Sima de los Huesos (originals):

AT-792, AT-1465, AT-1467,

AT- 168, AT-1763, AT-1828,

AT-2, AT-221, AT-2386,

Homo neanderthalensis $(\mathrm{n}=13) \quad$ AT-277, AT-2780, AT-2787,

AT-28, AT-300, AT-3188,

AT-3942, AT-4101, AT-562,

Homo sapiens $(\mathrm{n}=37)$ and chronological span: Australopithecus (n $1 / 46$ ), Paranthropus (n $1 / 41$ ), Plio-Pleistocene Homo (n $1 / 46)$, Late Lower Pleistocene Homo (n $1 / 43$ ), African Middle Pleistocene Homo (n $1 / 45$ ), Homo antecessor (n $1 / 42$ ), European Middle Pleistocene Homo (n $1 / 423$ ), Homo neanderthalensis (n $1 / 413$ ), and Homo sapiens (n $1 / 437$ ) (Table 1). We analyze Homo anteces-sor separately from the rest of Late Lower Pleistocene speci-mens because their premolars display morphological differences from the other groups.

Images of each occlusal surface were taken with a Nikon D1H camera fitted with an AF Micro-Nikon 105 $\mathrm{mm}, \mathrm{f} / \mathrm{2} .8 \mathrm{D}$. The camera was attached to a Kaiser

Copy Stand Kit RS-1 with grid baseboard, column, and adjustable cam-era arm. A leveling device was used to ensure that the lens was parallel to the baseboard and the cemento-enamel junc-tion (CEJ). For maximum depth of field, we used an aper-ture of $f / 32$. The magnification ratio was adjusted to $1: 1$, and a scale was included in each photograph and placed par-allel to, and at the same distance from the lens as, the oc-clusal plane.

Because asymmetry in dental morphological traits tends to be minor (Trinkaus, 1978; Scott and Turner, 1997), only the right antimere was used in the analyses. In order to maximize sample sizes, when the right tooth was absent or damaged, the left tooth was mirror-imaged with Adobe Photoshop. Teeth with severe attritional wear and those where one or more landmarks could not be clearly identified were not used.

\section{Geometric morphometric methods}

At the core of geometric morphometrics is Kendall's (1977) definition of shape as "all geometric information that remains when location, orientation and scale have been filtered out of an object." Shape can be described by configurations of landmarks, which are points of correspondence between different objects that match between and within populations (Bookstein, 1991; O'Higgins, 2000; Zelditch et al., 2004). Landmarks have both coordinates and a biological significance (Bookstein, 1991) ( Fig. 1A).

Procrustes techniques use least square methods to superimpose a given structure (target) at its corresponding landmarks (by translation, rotation, and scaling) onto a reference structure (Bookstein, 1991) (Fig. 1B). In the Generalized Procrustes Analysis (GPA) (Rohlf and Slice, 1990), all specimens (many target configurations) are aligned to their mean shape (reference configuration). The results of the generalized Procrustes superimposition are scatters of corresponding landmarks (Procrustes shape coordinates) around their means (Fig. 1B,C). The shape of a GPA superimposed landmark configuration is defined by the entirety of its residual coordinates.

During the scaling procedure of GPA, a scaling factor called "centroid size" is obtained. It is defined as the square root of the summed squared distances between the centroid (the mean of all landmark coordinates of a specimen) and each of the landmarks. It is a powerful size measurement because it
14, DV 15 (originals)

Modern human collection from Institute of Anthropology of the University of Coimbra 


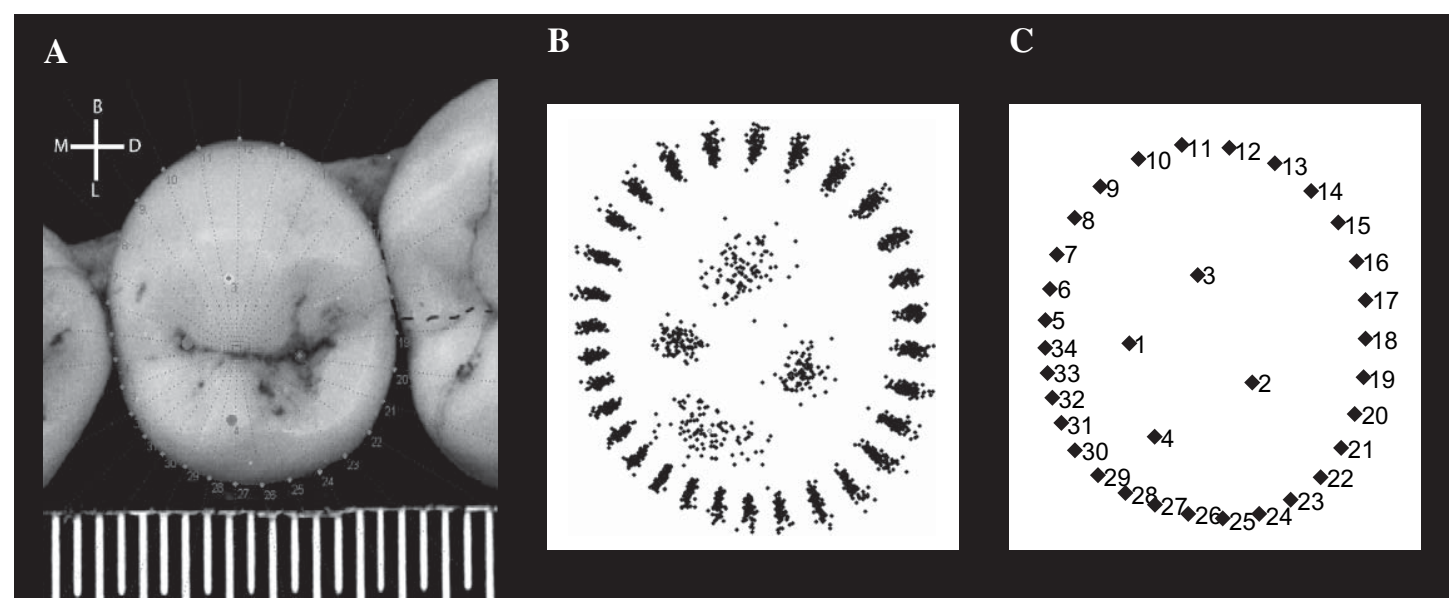

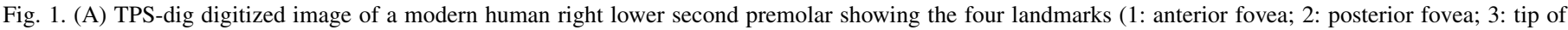

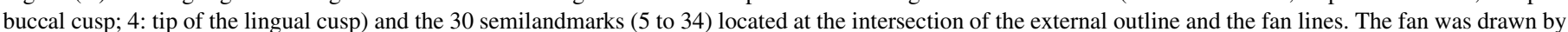

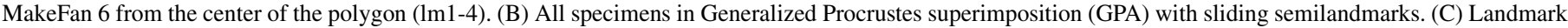
configuration of the mean shape of all specimens, also called "consensus" or "reference" shape.

does not induce a correlation between size and shape (Zelditch et al., 2004). Only in the case of allometry can correlations between size and shape (Bookstein, 1991) be observed.

Thin plate splines can be used to produce another category of shape variables, partial warps and uniform component scores (Bookstein, 1989, 1991). Thin plate splines (TPS) quantify the shape differences between two objects by a Procrustes fit of both, and by a deformation of the first specimen into the second one. This deformation consists of a uniform component (such as shearing) that describes uniform, general differences in shape, and a non-uniform component that describes localized differences in shape (Bookstein, 1991). The nonuniform component requires bending energy. The properties of this bending energy can be used to derive a set of powerful shape descriptors, the partial warps plus the uniform component (Bookstein, 1991, 1996; Rohlf, 1996).

The principal components of Procrustes shape data have been termed relative warps (Bookstein, 1991). These relative warps reflect principal patterns of shape variation and have recently found multiple applications in paleoanthropology (Bookstein et al., 1999; Rosas and Bastir, 2002; Bookstein et al., 2003; Bastir et al., 2004, 2005).

In the present context, geometric morphometrics overcome some methodological difficulties related to absolute toothorientation within the jaw and further helps us to understand outline shape variation with respect to biologically meaningful structures, such as the spatial configuration of cusps and fossae as well as their relationship to overall size.

\section{Landmarks}

Landmarks are defined by geometric characteristics of the hard and soft tissues they aim to describe (Bookstein, 1991). These geometric features may be seen as "internal criteria" such as suture crossings, bone intersections, bone processes, or other biologically meaningful features (Bookstein, 1991). Within and between populations, a landmark in one specimen corresponds geometrically and biologically to the same landmark in other specimens (Zelditch et al., 2004). Because landmarks are defined by the biological properties of their location itself, they carry relevant morphological information in all directions of their coordinate system. In $2 \mathrm{D}$, they have two well defined coordinates.

Here, landmarks were chosen because of their significance in assessing premolar variability (Biggerstaff, 1969) and their ease of identification in occlusal images. The occlusal morphology of a P4 consists of two main cusps and a talonid area. The median longitudinal fissure marks the boundary between the protoconid (buccal cusp) and the metaconid (lingual cusp). The distobuccal fovea/transverse fissure and its intersection with the buccal and lingual borders of the crown delimit the talonid area. Relative to these features, we define the following landmarks (Fig. 1):

- Landmark 1: Anterior/mesial fovea or the intersection of the medial longitudinal fissure with the mesial foveal fissure/transverse fissures.

- Landmark 2: Posterior/distal fovea or intersection of the medial longitudinal fissure with the distal foveal fissure/ transverse fissures.

- Landmark 3: The tip of the buccal cusp (or protoconid) determined by inspection. In the case of worn teeth, this point will be marked in the center of the dentinal facet.

- Landmark 4: The tip of the lingual cusp (or metaconid) determined by inspection. In the case of worn teeth, this point is taken in the center of the dentinal facet.

For landmarks 1 and 2, if the anterior and posterior fovea are extremely reduced in the shape of a short transverse fissure (Genet-Varcin, 1962) or are absent, the points were marked at the extremes of the median longitudinal fissure as illustrated in Figure 1A. If the location of the landmarks was not clear, either in fossil or modern human teeth, the specimen was not included for analysis.

Landmarks 3 and 4 were located in one of two ways. When using a cast, the tips of the main cusps were marked on the 
cast with soft pencil prior to photographing. When using an original or when permission to mark was denied, the tips of the main cusps were visually located in the images by simultaneously examining the fossil. Seventy percent of our sample is based on examination of original fossils (Table 1), undoubtedly helpful for a precise identification of the landmarks in the picture. When the tooth showed little wear, the cusp tip was marked in the center of the wear facet.

When mesial and/or distal borders of the teeth were affected by light interproximal wear, original borders were estimated by reference to the overall crown shape and the buccolingual extent of the wear facets, following Bailey and Lynch (2005) (Fig. 2).

\section{Sliding semilandmarks}

For the assessment of occlusal outlines, no landmarks are available. However, semilandmarks can be used for this purpose. Semilandmarks have one welldefined coordinate (putting them on the curve), while the other is arbitrary. There-fore, semilandmarks have been characterized as mathemati-cally "deficient" to some degree (Bookstein, 1991, 1997; Gunz et al., 2005). Only their information regarding the shape of the outline is biologically and statistically useful. Their rel-ative spacing along this outline is not relevant because this does not modify the shape of the curve. Therefore, they have reduced degrees of freedom (Zelditch et al., 2004).

"Sliding techniques" can be used to minimize the effects of the arbitrary placement along the curve (Bookstein, 1996, 1997; Bookstein et al., 2002; Gunz et al., 2005). In applying these methods, a "semilandmark" is converted into a "sliding semilandmark." Sliding semilandmarks are intended to describe the shape of an outline. However, the actual shape of the curve is not known because it consists of many more land-marks than are usually digitized. Thus, this shape is approxi-mated by the use of tangents to the semilandmarks. The tangent is approximated by a parallel to a line connecting the neighbor points of the semilandmark under consideration.

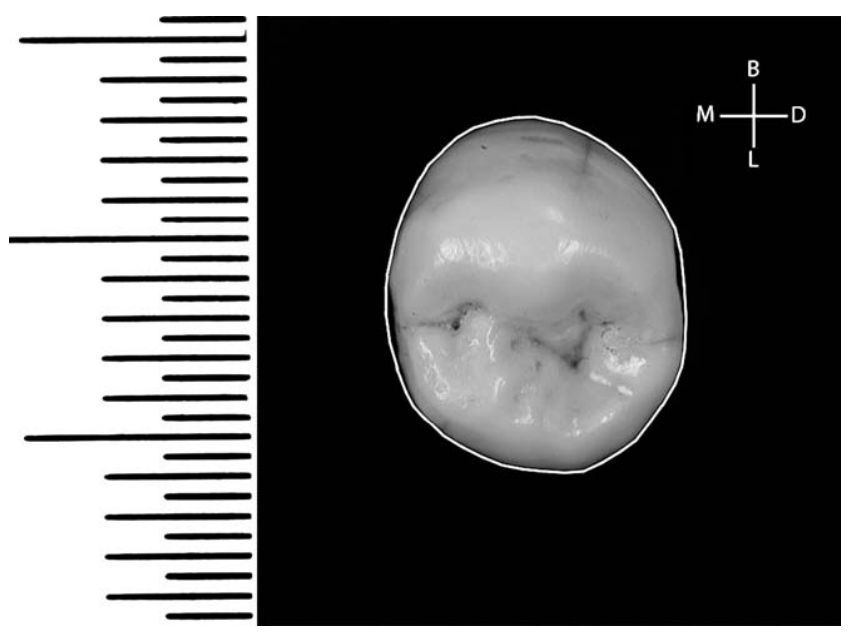

Fig. 2. Sima de los Huesos right lower second premolar corrected for interproximal wear.
Sliding semilandmarks are then recalculated to move ("slide") along the curve. Actually, and for reasons of optimizing the shape information, the semilandmark slides along its tangent to the outline until its final position minimizes one of several possible criteria, such as the bending energy (Bookstein, 1997; Bookstein et al., 2002, 2003; Mitteroecker et al., 2004; Bastir et al., in press), the distances between semi-landmarks between reference and target (Sheets et al., 2004), or the Procrustes distance between reference and target (Rohlf, 1998a). Each of these criteria have different advantages and disadvantages (Sheets et al., 2004; Zelditch et al., 2004; Bastir et al., in press). Here we use sliding semilandmarks until Pro-crustes distance is minimized between the reference and the target (Rohlf, 1998a). This procedure includes the uniform and nonuniform part of shape variation, although to our knowledge no study has yet worked with this approach. However, it has been pointed out that this criterion should be of particular interest in phylogenetic and systematic questions (Gunz et al., 2005; Gunz, pers. comm.; Rohlf, pers. comm.).

It is important to note that although sliding semilandmarks do not provide homologous points, if adequately computed, they can provide comparable points of homologue structures for the sample under study. Because the use of sliding semi-landmarks is relatively new, few studies have been published to date dealing differently with this issue (Bookstein, 1997; Rohlf, 1998a; Bookstein et al., 1999, 2002; Mitteroecker et al., 2004; Sheets et al., 2004; Bastir et al., in press). In its favor, the sliding technique provides non-arbitrary criteria (Zelditch et al., 2004; Gunz et al., 2005) and allows curves or outlines to be analyzed within the Procrustes scheme of shape analysis (Rohlf and Slice, 1990; Slice, 2001; Rohlf, 2003). However, it is important to keep in mind that no stan-dardized protocol has yet been developed for the use of sliding semilandmarks, and further research needs to be done to ad-dress this.

\section{Repeatability and statistical analysis}

Possible measurement error tied to the digitizing process was assessed by repeating the complete data recording procedure of a random subsample of five specimens. TPS-Util software (Rohlf, 1998b) was used to randomize the order of the data by the computer, and the first five specimens of the random data set were selected. This random test-sample consisted of two modern humans (M-33, $\mathrm{MH}-84)$ and three fossils (Krapina 50, Krapina E, AT-277). These data were analyzed by several methods. First, a principal component analysis (PCA) of the data was performed for visual inspection of the scatters. The results of this PCA are shown in Figure 3. Second, Matrix cor-relations [Mantel tests, (Mantel, 1967)] of Procrustes distance matrices were used to calculate all possible correlations between the different days of measurement. These correlations varied between 0.994 and 0.998 (mean $r 1 / 40.996$ ). Finally, we used Disparity Box software (Zelditch et al., 2003) to compare variation (measured as Procrustes distances) within each tooth (due to repeated measurement), with total variation in the 


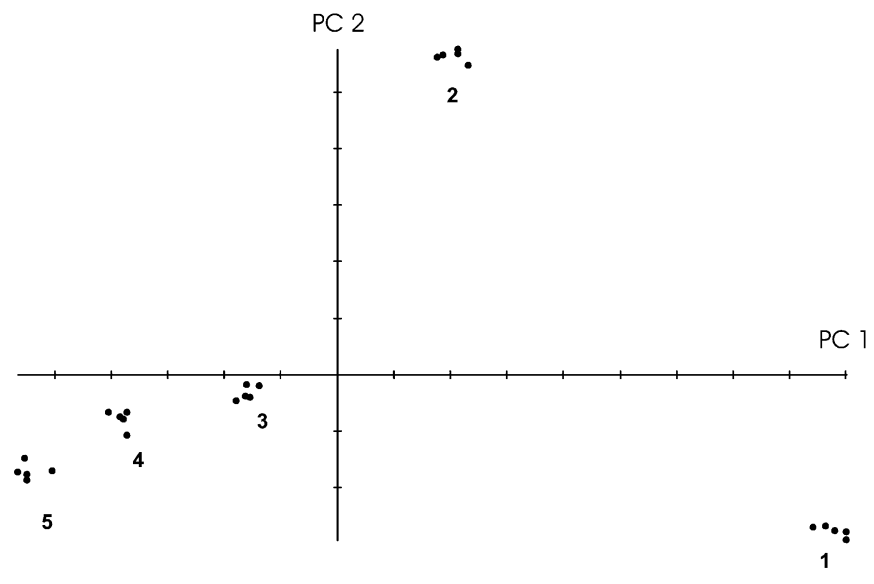

Fig. 3. Principal components I and II for repeatability analysis. Note that the data scatter much closer within specimens than between specimens.

random sample. This analysis showed that the repeated measures of variation of a single tooth were $1.8 \%$ of the total variation appearing in the random sample.

Since no normal distribution of shape data can be assumed using sliding semilandmarks, data ordination such as principal component analysis and permutation tests have been suggested instead of parametric statistics (Gunz et al., 2005). Therefore, we used principal component analysis [relative warps analysis; (Bookstein, 1991; Rohlf, 1993)] of the partial warp and uniform components scores $(\alpha=0)$ (Rohlf, 1996, 1998a; Rohlf et al., 1996; Bastir et al., 2005) to assess general patterns of variation. In addition, we used multivariate regression analysis (Rohlf, 1996, 1998b) of shape on size accompanied by permutations $(n=10000)$ to test for allometry. All the results are visualized by thin plate splines.

To help understand these results obtained with the novel application of the Procrustes techniques, we have also measured the occlusal polygon area by classical morphometric methods. With the AnalySIS ${ }^{\circledR}$ software, we measured from the occlusal photograph the total area of the crown (AC) and the occlusal polygon area (OPA), defined by the union of the four landmarks. We obtained the relative occlusal polygon area by dividing $\mathrm{AC} /$ OPA. Inter-group comparisons with a one-way ANOVA posthoc test (LSD or least significant distance) were performed.

\section{Results}

\section{Principal component analysis}

The results of the principal component analyses are shown in Figure 4 and Table 2. The first two factors account for $48 \%$ of the total variation (PC I: $29.7 \%$ and PC II: 18.3\%). Morphological variation along PC I is clearly illustrated by the TPSgrids of the relative warps in the extremes of the $\mathrm{x}$-axis (Fig. 4). Specimens with positive PC I values tend to display a circular shape, whereas premolars plotted in the left half of the graph show a more elongated outline with a distolingual bulge. Among the intracrown landmarks, protoconid and metaconid (landmarks 3 and 4) are the most variable points. The line connecting protoconid and metaconid shortens and moves from a buccal-mesiolingual diagonal direction to a more verticalized alignment. Specimens with positive loadings for PC I have a metaconid that is either distal or central relative to the protoconid, whereas premolars in the left side of the graph have a more mesial metaconid. Along the PC I axis, the protoconid moves progressively towards the lingual border, and the occlusal polygon (defined by the four landmarks) decreases in area and assumes a more exocentric position, closer to the lingual crown border. The relative area of the lingual portion of the tooth (delimited by the median longitudinal fissure and its prolongation to the borders of the crown) decreases relative to the buccal portion in specimens with positive loadings on PC I. On the contrary, teeth holding negative PC I values possess a lingual segment that is equal to, or relatively larger than, the buccal counterpart.

The morphological changes linked with PC II are more subtle than those of PC I. Along the PC II axis, an imaginary line connecting the protoconid and the metaconid is positioned more distally at higher loadings, particularly due to the position of the buccal cusp. The occlusal polygon becomes more centrically located and relatively larger as the PC II loading increases. The external contour tends to be distolingually enlarged the more negative the PC II value.

Although there is a considerable overlap among species, negative loading on PC I is mainly shown by Australopithecus and early Homo specimens, whereas specimens from all groups can be found in the central area. The positive extreme of PC I is primarily occupied by modern humans. These results suggest that the primitive pattern of mandibular second premolar occlusal morphology is an elongated (subrectangular) outline with a relatively larger occlusal polygon and a mesially displaced metaconid. This is the shape displayed by all the australopithecines except AL-266 (which still is very close to the zero value). Clustered with Australopithecus $\mathrm{P} 4 \mathrm{~s}$ are all the Plio-Pleistocene and the late Lower Pleistocene Homo specimens, the Paranthropus specimen, and four of the five African Middle Pleistocene specimens. This pattern contrasts with a centered metaconid and a more circular outline. This is the pattern displayed by $70 \%$ of the Homo sapiens sample and nearly exclusive to this taxon. The teeth belonging to $H$. heidelbergensis (European Middle Pleistocene hominins) and $H$. neanderthalensis are variable in their distribution $(43.4 \%$ and $69 \%$ of these taxa, respectively, display negative PC I values).

Analyzing the distribution of the specimens along PC II, representatives from nearly all species are found in the lower half of the graph, whereas late Lower Pleistocene representatives fall only in the upper left quadrant of the graph (Fig. 4). Despite belonging to the same time range, Homo antecessor is isolated in the lower right quadrant. Negative PC II values isolate Australopithecus in the lower half of the graph and, with one exception (AL-266), they are all clustered in the left lower quadrant. Only Homo sapiens and some Homo heidelbergensis specimens have positive PC I and PC II loadings. When the specimens are arranged chronologically, it seems that distal displacement of the protoconid (recorded in the PC II) appears phylogenetically earlier than the metaconid displacement (recorded in the PC I). 


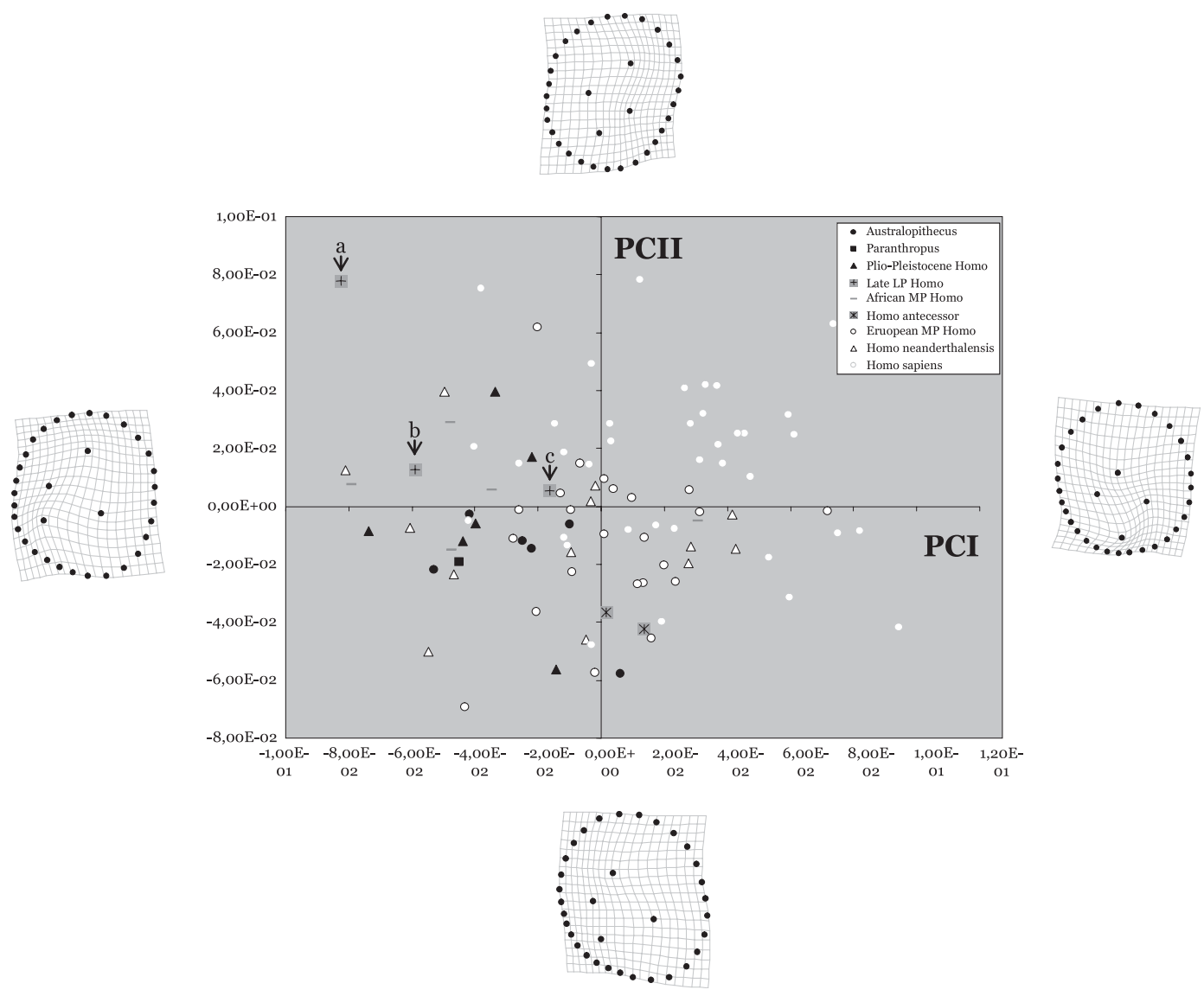

Fig. 4. Projection of individual P4 crowns on PCI and PCII. In the extremes of the axis, TPS-grids illustrate the morphological variation trends of the specimens along each principal component. These grids show how a TPS transformation of the mean shape into a theoretical specimen would look if its PC-score were at an extreme point of the one PC axis and zero at all other axes. The arrows point to: a) KNM-ER 992, b) Sangiran 6, c) KNM-WT 15000, mentioned in the text.

The trend in the OPA reduction, ascertained by the geometric morphometric analysis along the PC I axis, can be read in Table 3. Australopithecus, the Plio-Pleistocene hominins, and the single Paranthropus specimen show the highest values for the relative occlusal polygon area, whereas late Homo species exhibit the lowest values. The small $\mathrm{n}$ of some of the groups precludes statistical comparison among all groups, but Australopithecus is significantly different from Homo heidelbergensis, Homo neanderthalensis, and Homo sapiens $(\mathrm{p}<0.05)$. The

Table 2

Relative Warps (RW) analysis. The table displays the first ten principal components, the singular values, and the percentage of explained variance

\begin{tabular}{lccc}
\hline RW & $\begin{array}{c}\text { Singular } \\
\text { value }\end{array}$ & $\begin{array}{c}\text { \% explained } \\
\text { variance }\end{array}$ & $\begin{array}{c}\text { \% cumulative } \\
\text { variance }\end{array}$ \\
\hline 1 & 0.38 & 29.65 & 29.65 \\
2 & 0.29 & 18.30 & 47.95 \\
3 & 0.27 & 15.45 & 63.41 \\
4 & 0.19 & 7.35 & 70.76 \\
5 & 0.16 & 5.62 & 76.38 \\
6 & 0.15 & 4.95 & 81.33 \\
7 & 0.13 & 3.88 & 85.21 \\
8 & 0.12 & 3.03 & 88.24 \\
9 & 0.11 & 2.70 & 90.94 \\
10 & 0.09 & 1.65 & 92.59 \\
\hline
\end{tabular}

Plio-Pleistocene specimens are also significantly different from Homo heidelbergensis and Homo sapiens.

\section{Regression analysis}

Permutation tests $(n=10000)$ of multivariate regression analysis of partial warps and uniform components scores on centroid size indicate a slight but significant $(\mathrm{P}=0.000)$ allometry that accounts for $7.5 \%$ of overall variation. The

Table 3

Relative occlusal polygon areas calculated for the studied groups

\begin{tabular}{lcc}
\hline & $\mathrm{n}$ & $\begin{array}{c}\text { Mean } \pm \text { Std. } \\
\text { deviation }\end{array}$ \\
\hline Australopithecus & 6 & $13.59 \pm 1.93$ \\
Paranthropus & 1 & 17.80 \\
Plio-Pleistocene Homo & 6 & $13.24 \pm 3.54$ \\
Late Lower Pleistocene Homo & 3 & $12.50 \pm 2.30$ \\
H. antecessor & 2 & $11.50 \pm 2.39$ \\
Middle Pleistocene Homo & 5 & $12.69 \pm 2.54$ \\
H. heidelbergensis & 23 & $10.94 \pm 1.92$ \\
H. neanderthalensis & 13 & $11.27 \pm 2.64$ \\
H. sapiens & 37 & $10.95 \pm 1.87$ \\
\end{tabular}


way this size component would contribute to the shape variation is illustrated in Figure 5: smaller premolars tend to be circular in outline with the intra-crown structures centrally located. Larger premolars tend to display subrectangular outlines with an enlarged lingual surface and mesially displaced intracrown structures.

\section{Discussion}

Our analysis reveals that although mandibular P4 crown shape is polymorphic, a primitive-derived gradient is apparent in the hominin fossil record. It is possible to distinguish typical patterns of $\mathrm{P} 4$ shape for particular groups such as Homo sapiens or Australopithecus. The TPS-grids data (Fig. 4) suggest that there is a relationship between the external occlusal outline and the internal arrangement of the cusps. A mesially displaced metaconid is associated with an asymmetrical outline, whereas a symmetrical contour is often associated with a central lingual cusp. Thus, asymmetry is related to the existence of a bulging distolingual talonid which, in turn, is responsible for the mesial location of the metaconid. We interpret this morphology as the generalized primitive pattern. Dental reduction throughout the Pleistocene proceeded from this pattern.

\section{P4s external contour: asymmetry assessment}

Symmetrical teeth are described as those in which the buccal and lingual halves mirror one another. Bailey and Lynch (2005), using Homo erectus as an outgroup, concluded that symmetry rather than asymmetry is the primitive state. Thus, asymmetry would be an unevenness of the lingual contour derived from a truncated mesiolingual lobe and would likely represent a derived condition in Neandertals (Bailey, 2002a; Bailey and Lynch, 2005). Our study confirms that asymmetry is the norm for Homo neanderthalensis ( $70 \%$ of our sample), but we do not consider this asymmetry derived. The apparent discordance in assessing the polarity of P4 asymmetry between these studies may derive from the sample employed to assess the primitive

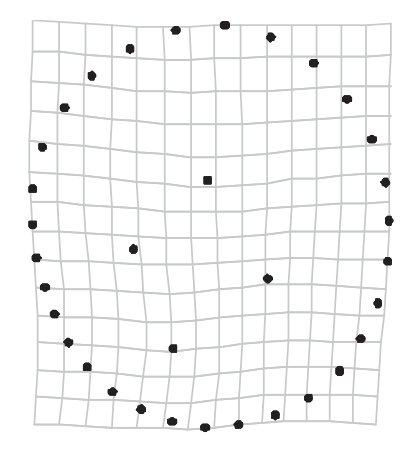

Small ( $C S=1.72)$

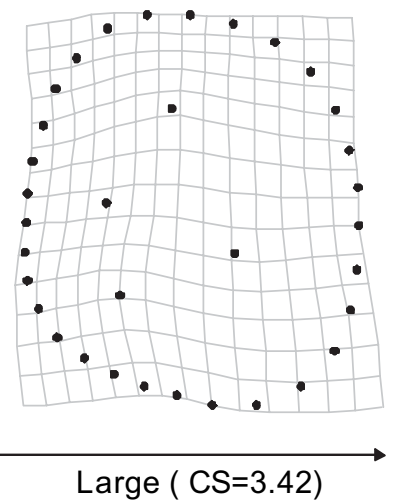

Fig. 5. Multivariate regression analysis. The TPS-grids illustrate the morphological variants related to variation in Centroid Size. The TPS- grid shows the transformation of the mean shape into a small individual (left), as estimated by the regression model, and into a large specimen (right). state. Bailey and Lynch (2005) included Zhoukoudian premolars as part of their Homo erectus outgroup. On average, these might look more oval (Kaifu et al., 2005) than the H. erectus from Sangiran included in this study (Fig. 6). The composition of Homo erectus is a controversial issue (e.g., Aguirre and de Lumley, 1977). In addition, some researchers have recently argued that the Zhoukoudian H. erectus crania are more derived than those from Indonesia or Africa (Antón, 2002; Kidder and Durband, 2004); potentially, this could apply to their teeth as well. Whatever the influence of our different $H$. erectus samples on P4 morphology, we also used Australopithecus and early Homo to help determine the polarity of P4 crown asymmetry. All of our earlier groups suggest that P4 asymmetry is the primitive condition. Another reason for the discordance between these studies may relate to the axis used to measure the asymmetry. In all teeth, and especially when dealing with isolated teeth, the orientation of the tooth when identifying the mesiodistal and buccolingual axes may vary. Thus, a certain degree of variability in the location of the plane through which we would assess the asymmetry of the contour may exist. This problem is overcome in the case of the geometric morphometric landmarks because they are biologically well-defined structures in the tooth, regardless of the orientation of the tooth. Procrustes superimposition orients all the premolars in a homologous way. In addition, sliding semilandmarks spot geometrically comparable points along the outline of the crown, so the specific area responsible for shape differences among groups can be objectively localized.

We believe that the "unevenness" of the lingual half of the crown in Neandertals is mainly due to an expansion of the distolingual portion rather than to a moiety of the mesiolingual part (Bailey and Lynch, 2005). The distal extension of the talonid leaves the intracrown structures in a mesial position within the tooth outline. The mesial displacement of both cusps has previously been described as a primitive arrangement typical of australopithecines, "arcanthropines," and some Neandertals (Genet-Varcin, 1962, 1966). In modern humans, the protoconid and metaconid occupy a more central location due to the reduced size of the distal talonid more than to the migration of the cusps (Genet-Varcin, 1962).

\section{P4 internal morphology: cusp configuration and the occlusal polygon}

The hominin fossil record provides clear evidence of size and shape changes of P4 within the genus Homo. The deciduous and permanent dentitions of modern humans are reduced compared to early Homo species (Wolpoff, 1971; Wood and Abbott, 1983; Bermúdez de Castro, 1993; Bermúdez de Castro and Nicolás, 1995). It has been suggested that different evolutionary lineages of Homo (e.g., modern human and Neandertal lineages) shared a similar pattern of reduction, not only in the global decrease in tooth size, but also in the sequential disappearance of cusps (Bermúdez de Castro, 1993; Bermúdez de Castro and Nicolás, 1995). In contrast, the anterior teeth have retained their relatively large size, even increasing in 


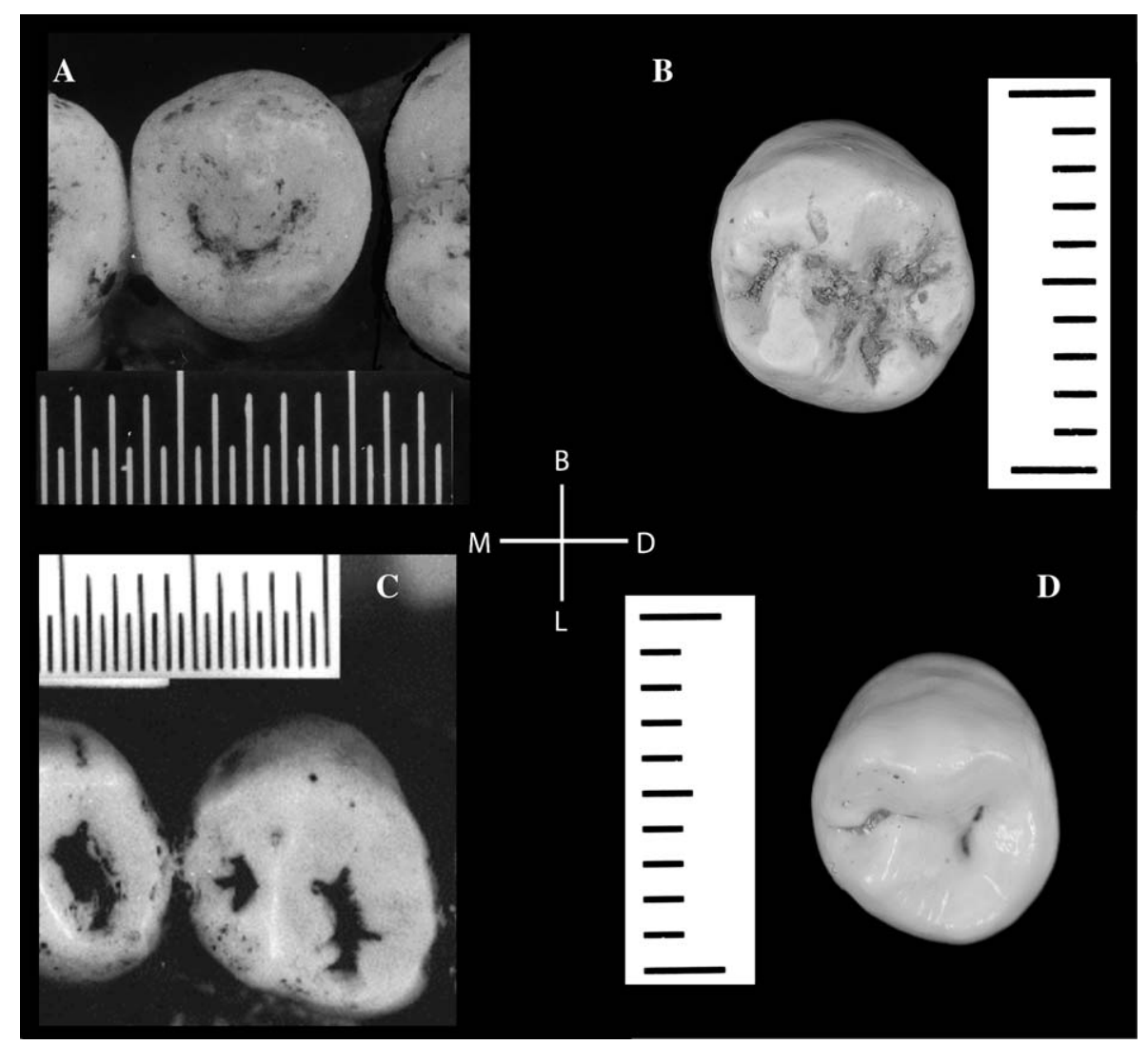

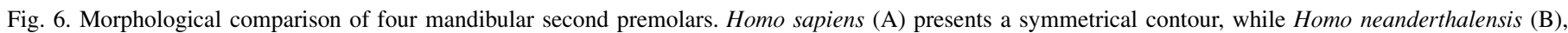

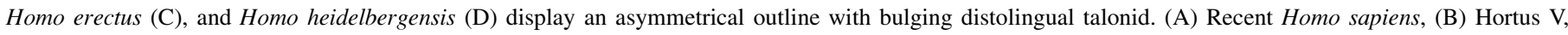
mirrored image, (C) Sangiran 6, (D) AT-3942.

some dimensions, as in Neandertals (Bermúdez de Castro, 1993). The imbalance between the sizes of I1-P3 and P4M3 in the $H$. heidelbergensis sample (Bermúdez de Castro, 1993; Bermúdez de Castro et al., 1999) suggests that different evolutionary trends have affected anterior and posterior dentitions. Thus, different biological processes may also be responsible for these trends.

In the later stages of hominin evolution, $\mathrm{P} 4$ size reduction begins with the reduction of the talonid. The distobuccal accessory cusps are the first to disappear in the fossil record, followed by the distolingual cusps (Genet-Varcin, 1962); distribution of specimens along PC II supports this idea (Fig. 4). The reduction of the buccal portion of the talonid, which is typically broad in Australopithecus, is probably responsible for the protoconid's "migration" to a more central position in the late Lower Pleistocene and some Middle Pleistocene Homo specimens (Fig. 4). The distolingual cusps together with the buccal talonid may contribute to an apparently more "squared" outline in some early Homo specimens (like KNM-ER 992 or KNM-WT 15000) when compared to others such as Sangiran 6 from Java. Nonetheless, the large talonids and the wide occlusal polygon cluster these specimens of early Homo in the upper left quadrant of the graph (see Fig. 4).

The development of the talonid together with its extra cusps has functional consequences for mastication. Selection appears to have favoured larger occlusal areas in Pliocene hominins
(Townsend et al., 1990; Suwa et al., 1996; McCollum and Sharpe, 2001). Interestingly, the modifications in the internal occlusal arrangement through the Pleistocene are generally related to a decrease in the occlusal polygon area (Table 3). As we can observe in the TPS-grids along the PCA graph (Fig. 4), the occlusal polygon is relatively large in Australopithecus and some early Homo specimens relative to H. sapiens. The lingual half of the crown of P4s tends to be subequal in size with, or even larger than, the buccal portion. Thus, the functional participation of the occlusal surface would be maximized in Pliocene and African Lower and Middle Pleistocene specimens.

In contrast, European Lower and Middle Pleistocene specimens (H. antecessor, H. heidelbergensis, and H. neanderthalensis) show a reduced occlusal polygon, perhaps related to the diminution of the lingual portion (see the TPS-grids of Fig. 4). In Homo sapiens, the reduced occlusal polygon occupies a more lingual location. Given the phylogenetic order of appearance of each cusp in the hominoid dentition (Gregory, 1916), later developing elements (such as the metaconid and other lingual cusps) are more prone to be affected by timing and spacing changes in the ontogenetic sequence (Mizoguchi, 1983; Macho and Moggi-Cecchi, 1992). The TPS-grids in the extremes of the PC I (Fig. 4) suggest that there is a relative diminution of the lingual portion of the $\mathrm{P} 4$ crown in later Homo specimens. It is not only $H$. neanderthalensis that is affected by this reduction process (cf. Bailey and Lynch, 2005), but 
also $H$. antecessor, and some specimens assigned to $H$. sapiens, $H$. neanderthalensis, and $H$. heidelbergensis. If the "mesial truncation" of the lingual crown is a consequence of a narrower lingual portion (Bailey and Lynch, 2005), the same pattern would be expected in $H$. sapiens. However, modern humans typically display a symmetrical P4 contour (Fig. 6).

We suggest that the reduced occlusal polygon of $H$. heidelbergensis/neanderthalensis is "compensated" for by the addition of extra lingual cusps and an enlarged talonid. Such compensation may indicate a biomechanically demanding diet, which might be supported by work on the anterior dentition that suggests Neandertals experienced heavy paramasticatory loads (Brace, 1963; Smith, 1983; Rak, 1986; Trinkaus, 1987; Demes and Creel, 1988) or intense, repetitive occlusal loads (O'Connor et al., 2003). In the case of the anterior dentition, selection favored a dental morphology better adapted to a biomechanically demanding diet (Molnar, 1971; Y'Edinak, 1978), and this could also apply to posterior dentition, despite its size reduction. Our findings are consistent with the hypothesis that Neandertals are the result of a process of character accretion which started during the Middle Pleistocene (Arsuaga et al., 1997; Hublin, 1998). In this view, some of the morphological traits considered to be typical of Neandertals are not Neandertal apomorphies but instead are distinctive combinations of symplesiomorphic characters (Patte, 1962; Franciscus and Trinkaus, 1995). For example, we find that $H$. heidelbergensis/neanderthalensis apparently show a distinctive combination of primitive dental traits (asymmetrical contour, mesially displaced metaconid, and extra lingual cusps) in association with other traits like a well-developed transverse ridge (Bailey, 2002a,b; Bailey and Lynch, 2005) (Fig. 6). This conformation of traits, in association with an exocentric position for the occlusal polygon, is first seen in one of the specimens assigned to $H$. antecessor, perhaps the last common ancestor of $H$. sapiens and $H$. neanderthalensis (Bermúdez de Castro et al., 1997). None of these traits are derived, since we see them in earlier specimens (see asymmetry and transverse ridge in Sangiran 6) (Fig. 6), but the particular combination seems only to be seen in high frequencies in Neandertal species and very rare in modern human samples (Bailey and Lynch, 2005). Thus, the P4 crown morphology of $H$. antecessor is consistent with it being the primitive condition from which the later Homo species pattern derived.

\section{Allometry}

Despite previous assumptions that $\mathrm{P} 4$ crown morphology is not influenced by size (Bailey and Lynch, 2005), our study finds that allometric variation accounts for $7.5 \%$ of the observed variation (Fig. 5). The allometric factor may be particularly driven by species in the extremes of teeth size variation. Large-toothed genera such as Australopithecus and Paranthropus (Wood and Uytterschaut, 1987) have subrectangular or ovoidal P4s (Fig. 6) with a disto-lingual bulge and mesially displaced main cusps (Genet-Varcin, 1962; Wood and Uytterschaut, 1987; Townsend et al., 1990; Suwa et al., 1996). In contrast, modern humans have small P4s (Wood and Abbott, 1983; Bermúdez de Castro, 1993; Bermúdez de Castro and Nicolás, 1995) with more circular outlines and more centrically located main cusps (Genet-Varcin, 1962).

\section{Conclusions}

The outline and the internal arrangement of hominid P4 crowns are polymorphic traits. Although P4 morphology provides little ability to correctly assign an isolated specimen to its corresponding species, it is possible to characterize the typical P4 pattern of a hominin group. The use of geometric morphometric methods overcomes the problem of general orientation when dealing with isolated teeth. The external contour of the P4 is related to cuspal morphology and slightly influenced by the overall size of the crown. The primitive pattern appears to be an asymmetrical outline with a mesially displaced metaconid, an extended talonid, and a broad occlusal polygon. The general trend of dental reduction throughout the Pleistocene has produced different morphological variants with a narrower occlusal polygon. Homo heidelbergensis/ neanderthalensis show a distinctive combination of plesiomorphic traits (asymmetry, mesial metaconid, and extended talonid), whereas modern humans evolved a more circular outline with a centrally located metaconid and reduced talonid.

Given the enormous potential of the methodology employed in this study, future research should explore larger dental samples and other dental classes, searching for taxonomic, phylogenetic, and developmental implications. It would also be very interesting to work in quantification methods for the correlation between internal arrangement and external shape.

\section{Acknowledgements}

We are grateful to all members of the Atapuerca research team. Special thanks to the Sima de los Huesos excavation team for their arduous and exceptional contribution. We also thank D. Lordkipanidze and A. Vekua from the Georgian National Museum; C. Bernis and J. Rasco'n from Universidad Auto'noma de Madrid; A. Pe'rez-Pe'rez, J. Galbany, F. Estebar-anz, and L.M. Martı'nez from the Universitat de Barcelona; E. Cunha from Universidade de Coimbra; J. Svoboda and M. Oliva from the Institute of ArchaeologyPaleolithic and Paleoethnol-ogy Research Center, Dolnı' Vestonice, Czech Republic; H. and M. A. de Lumley from Institut de Pale'ontologie Humaine, Paris; P. Tassy from Muse 'um national d'Histoire Naturelle, Paris; and I. Tattersall, K. Mowbray, and G. Sawyer from the American Museum of Natural History, New York; for providing access to the studied material and their helpful assistance when examining it. We thank James Rohlf at SUNY, Stony Brook, Dave Sheets at Canisius College, SUNY Buffalo, and Phillip Gunz and Phillip Mitteroecker at University of Vienna for dis-cussion and advice regarding some methodological aspects. This research was supported by funding from the Direccio'n General de Investigacio'n of the Spanish M. E. C., Project No. BOS2003-08938-C03-02 (2004e2007), the CSIC "Uni-dades Asociadas" Program, Spanish Ministry of Science and 
Education, Fundación Atapuerca, Fundación Duques de Soria, and Fundación Caja Madrid. Fieldwork at Atapuerca is supported by Consejería de Cultura y Turismo of the Junta de Castilla y León. This research was partly carried out under the Cooperation Treaty between Spain and the Republic of Georgia, hosted by the Fundación Duques de Soria and the Georgian National Museum.

\section{References}

Aguirre, E., de Lumley, M.-A., 1977. Fossil men from Atapuerca, Spain: their bearing on human evolution in the Middle Pleistocene. J. Hum. Evol. 6, 681-688

Antón, S.C., 2002. Evolutionary significance of cranial variation in Asian Homo erectus. Am. J. Phys. Anthropol. 118, 301-323.

Arsuaga, J.L., Martínez, I., Gracia, A., Lorenzo, C., 1997. The Sima de los Huesos crania (Sierra de Atapuerca, Spain). A comparative study. J. Hum. Evol. 33, 219-281.

Bailey, S.E., 2002a. A closer look at Neanderthal postcanine dental morphology: the mandibular dentition. Anat. Rec. New. Anat. 269, 148-156.

Bailey, S.E., 2002b. Neandertal dental morphology: implications for modern human origins. Ph.D. Dissertation, Arizona State University, Temple.

Bailey, S.E., Lynch, J.M., 2005. Diagnostic differences in mandibular P4 shape between Neandertals and anatomically modern humans. Am. J. Phys. Anthropol. 126 (3), 268-277.

Bastir, M., Rosas, A., Kuroe, K., 2004. Petrosal orientation and mandibular ramus breadth: evidence of a developmental integrated petroso-mandibular unit. Am. J. Phys. Anthropol. 123, 340-350.

Bastir, M., Rosas, A., Sheets, D.H., 2005. The morphological integration of the hominoid skull: a Partial Least Squares and PC analysis with morphogenetic implications for European Mid-Pleistocene mandibles. In: Slice, D. (Ed.), Modern Morphometrics in Physical Anthropology. Kluwer Academic/Plenum Publishers, New York.

Bastir, M., Rosas, A., O'Higgins, P. Craniofacial levels and the morphological maturation of the human skull. J. Anat., in press.

Bermúdez de Castro, J.M., 1993. The Atapuerca dental remains: new evidence (1987-1991 excavations) and interpretations. J. Hum. Evol. 24, 339-371.

Bermúdez de Castro, J.M., Nicolás, M.E., 1995. Posterior dental size reduction in hominids: the Atapuerca evidence. Am. J. Phys. Anthropol. 96, 335-356.

Bermúdez de Castro, J.M., Rosas, A., Nicolás, M.E., 1999. Dental remains from Atapuerca-TD6 (Gran Dolina site, Burgos, Spain). J. Hum. Evol. $37,523-566$.

Bermúdez de Castro, J.M., Arsuaga, J.L., Carbonell, E., Rosas, A., Martínez, I., Mosquera, M., 1997. A hominid from the Lower Pleistocene of Atapuerca, Spain: possible ancestor to Neandertals and modern humans. Science 276, 1392-1395.

Biggerstaff, R.H., 1969. The basal area of posterior tooth crown components: the assessment of within tooth variation of premolars and molars. Am. J. Phys. Anthropol. 31, 163-170.

Bookstein, F.L., 1989. Principal warps: thin-plate splines and the decomposition of deformations. IEEE Trans. Pattern Anal. Mach. Intell. 11, 567-585.

Bookstein, F.L., 1991. Morphometric Tools for Landmark Data. Cambridge University Press, Cambridge.

Bookstein, F., 1996. Applying landmark methods to biological outline data. In: Mardia, K.V., Gill, C.A., Dryden, I.L. (Eds.), Image Fusion and Shape Variability Techniques. Leeds University Press, Leeds.

Bookstein, F.L., 1997. Landmark methods for forms without landmarks: morphometrics of group differences in outline shape. Med. Image Anal. 1, 225-243.

Bookstein, F., Schäfer, K., Prossinger, H., Seiderl, H., Fieder, M., Stringer, C., Weber, G.W., Arsuaga, J.L., Slice, D.E., Rohlf, F.J., Recheis, W., Mariam, A.J., Marcus, L.F., 1999. Comparing frontal cranial profiles in archaic and modern Homo by morphometric analysis. Anat. Rec. New. Anat. 257, 217-224.
Bookstein, F.L., Sampson, P.D., Connor, P.D., Streissguth, A.P., 2002. Midline corpus callosum is a neuroanatomical focus of fetal alcohol damage. Anat. Rec. New. Anat. 269, 162-174.

Bookstein, F.L., Gunz, P., Mitteroecker, P., Prossinger, H., Schaefer, K., Seidler, H., 2003. Cranial integration in Homo: singular warp analysis of the midsagittal plane in ontogeny and evolution. J. Hum. Evol. 44, 167-187.

Brace, C.L., 1963. Structural reduction in human evolution. Am. Nat. 97, 39-49.

Demes, B., Creel, N., 1988. Bite force, diet, and cranial morphology of fossil hominids. J. Hum. Evol. 17, 657-670.

Fraipont, J., Lohest, M., 1887. La race humaine de Néanderthal ou de Canstadt en Belgique. Arch. Biol. 7, 587-757.

Franciscus, R.G., Trinkaus, E., 1995. Determinants of retromolar space presence in Pleistocene Homo mandible. J. Hum. Evol. 28, 577-595.

Genet-Varcin, E., 1962. Évolution de la couronne de la seconde prémolaire inférieure chez les hominidés. Ann. Paleon (Vertebres) XLVIII, 59-81.

Genet-Varcin, E., 1966. Étude de dents permanentes provenant du gisement moustérien de La Croze del Dua. Ann. Paleon (Vertebres) LII (1), 6-28.

Gregory, W.K., 1916. Studies on the evolution of the Primates. I. The CopeOsborn 'theory of trituberculy' and the ancestral molar patterns of the Primates. Bull. Am. Mus. Nat. Hist. 35, 238-257.

Gunz, P., Mitteroecker, P., Bookstein, F.L., 2005. Semilandmarks in three dimensions. In: Slice, D. (Ed.), Modern Morphometrics in Physical Anthropology. Kluwer Academic/Plenum Publishers, New York, pp. 73-98.

Hublin, J.J., 1998. Paleogeography, and the evolution of the Neandertals. In: Akazawa, T., Aoki, K., Bar-Yosef, O. (Eds.), Neandertals and Modern Humans in Western Asia. Plenum Press, New York, pp. 295-310.

Irish, J.D., 1993. Biological affinities of late pleistocene through modern African aboriginal populations: the dental evidence. Ph.D. Dissertation, Arizona State University, Tempe.

Kaifu, Y., Baba, H., Aziz, F., Indriati, E., Schrenk, F., Jacob, T., 2005. Taxonomic affinities and evolutionary history of the early Pleistocene hominids of Java: dentognathic evidence. Am. J. Phys. Anthropol.

Kendall, D.G., 1977. The diffusion of shape. Adv. App. Probab. 9, 428-430.

Khul, F.P., Giardina, C.R., 1982. Elliptic Fourier analysis of a closed contour Comput. Graph. Imag. Proc. 18, 259-278.

Kidder, J.H., Durband, A.C., 2004. A re-evaluation of the metric diversity within Homo erectus. J. Hum. Evol. 46, 297-313.

Lestrel, P.E., 1974. Some problems in the assessment of morphological shape differences. Yearb. Phys. Anthropol. 18, 140-162.

Lestrel, P.E. (Ed.), 1997. Fourier Descriptors and Their Applications in Biology. Cambridge University Press, New York.

Ludwig, F.J., 1957. The mandibular second premolars: morphologic variation and inheritance. J. Dent. Res. 36 (2), 263-273.

Lumley, H., de Lumley, M.-A., de Brandi, R., Guerrier, E., Pillard, F., Pillard, B., 1972. La Grotte moustérienne de Hortus. Editions du Laboratoire de Paléontologie Humaine et de Préhistoire, Marseille.

Macho, G.A., Moggi-Cecchi, J., 1992. Reduction of Maxillary Molars in Homo sapiens sapiens: a different perspective. Am. J. Phys. Anthropol. 87, 151-159.

Mantel, N.A., 1967. The detection of disease clustering and a generalized regression approach. Cancer Res. 27, 209-220.

McCollum, M.A., Sharpe, P.T., 2001. Developmental genetics and early hominid craniodental evolution. Bioessays 23, 481-493.

Mitteroecker, P., Gunz, P., Bernhard, M., Schaefer, K., Bookstein, F.L., 2004 Comparison of cranial ontogenetic trajectories among great apes and humans. J. Hum. Evol. 46, 679-698.

Mizoguchi, Y., 1983. Influences of the earlier developing teeth upon the later developing teeth. Bull. Nat. Sci. Mus. Tokyo, Ser. D 9, 33-45.

Molnar, S., 1971. Human tooth wear, tooth function and cultural variability. Am. J. Phys. Anthropol. 34, 175-190.

O’Connor, C.F., Franciscus, R.G., Holton, N.E., 2003. Bite force production capability and efficiency in Neandertals and modern humans. Am. J. Phys. Anthropol. 127, 129-151.

O'Higgins, P., 2000. The study of morphological variation in the hominid fossil record: biology, landmarks and geometry. J. Anat. 197, 103-120. 
Patte, $E^{\prime}$., 1962. La dentition des Ne'anderthaliens. Masson et Cie, Pa $237 \mathrm{e} 242$.

Rak, Y., 1986. The Neanderthal: a new look at an old face. J. Hum.

Evo1516,164.

Rohlf, F.J., Slice, D., 1990. Extensions of the Procrustes method for aptemal superimposition of landmarks. Syst. Zool. 39, 40-59.

Rohlf, F.J., Loy, A., Corti, M., 1996. Morphometric analysis of old world Talpidae (Mammalia, Insectivora) using partial warp scores. Syst. Bio $45,344 \mathrm{e} 362$.

Rohlf, F.J., 1993. Relative warp analysis and an example of its apptricantitio wings. In: Marcus, L.F., Bello, E., ValdecasasGarcia, A.(Eds.), Contributions to Morphometrics. Museo Nacional de Ciencias Naturales, Consejo Superior De Investigaciones Cientificas, Madrid, pp. 131e159.

Rohlf, F.J., 1996. Morphometric spaces, shape components and the effects of linear transformations. In: Marcus, L.F. (Ed.), Advances in Morphometrics Plenum Press, New York, pp. 117-128.

Rohlf, F.J., 1998a. Tpsrelw. Ecology \& Evolution, SUNY, Stony Brook, New York.

Rohlf, F., 1998b. TpsUtil. Department of Ecology and Evolution, State University, Stony Brook, New York.

Rohlf, F., 1998c. Tpsregr. Department of Ecology and Evolution, State

Unisidy; Stony Brook, New York.

Rohlf, F.J., 2003. Bias and error in estimates of mean shape in geqphetrietrices: J. Hum. Evol. 44, 665e683.

Rosas, A., Bastir, M., 2002. Thin-plate spline analysis of allometry

anddsexudflism in the human craniofacial complex. Am. J. Phys. Anthropol.

$117,236 \mathrm{e} 245$.

Scott, G.R., Turner II, C.G., 1997. The Anthropology of Modern Human

Teeth: Dental Morphology and its Variation in Recent Human Populations. Cambridge University Press, Cambridge.

Sheets, D.H., Kim, K., Mitchel, C.E., 2004. A combined landmark and outline-

based approach to ontogenetic shape change in the Ordovician Trilobite Triarthrus Becki. In: Elewa, A. (Ed.), Morphometrics in Paleontology. Springer, Berlin, Wien.

Seiffert, E.R., Kappelman, J., 2001. Morphometric variation in the hominoid

orbital aperture: a case study with implications for the use of variable char-acters in Miocene catarrhine systematics. J. Hum.

Evol. 40, 301e318.

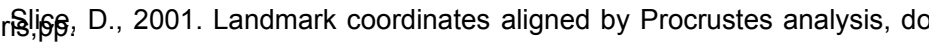
notlie in Kendall's shape space. Syst. Biol. 50, 141-149.

Smith, R.J., 1983. Behavioral interpretations of changes in craniofacial morphology across the archaic/modern Homo sapiens transition. BAR Int. Ser. 164, 137-209.

Suwa, G., White, T.D., Howell, F.C., 1996. Mandibular postcanine dentition from the Shungura Fomration, Ethiopia: crown morphology, taxonomic

Biol. locations, and Plio-Pleistocene hominid evolution. Am. J. Phys. Anthropol. 101, 247-282.

Townsend, G., Yamada, H., Smith, P., 1990. Expression of the entoconulid (sixth cusp) on mandibular molar teeth of an Australian Aboriginal population. Am. J. Phys. Anthropol. 82, 267-274.

Trinkaus, E., 1978. Bilateral asymmetry of human skeletal nonmetric traits. Am. J. Phys. Anthropol. 49, 315-318.

Trinkaus, E., 1987. The Neandertal face: evolutionary and functional perspecrics. tives on a recent hominid face. J. Hum. Evol. 16, 429-443.

Turner II, C.G., Nichol, C.R., Scott, G.R., 1991. Scoring procedures for key morphological traits of the permanent dentition: the Arizona State University dental anthropology system. In: Kelley, M., Larsen, C. (Eds.), Advances in Dental Anthropology. Wiley-Liss, New York, pp. 13-31.

Uytterschaut, H.T., Wood, B.A., 1989. Dental morphology: characterization and identification of Australopithecines and Homo habilis. In: Hominidae. Proceedings of the Second International Congress of Human Paleontology. Editoriale jaca Book Milan, pp. 183-188.

Wolpoff, M.H., 1971. Metric Trends in Hominid Dental Evolution. In: Studies in Anthropol. No. 2. Case Western Reserve Universty, pp. 1-244.

Wood, B.A., Abbott, S.A., 1983. Analysis of the dental morphology of PlioPleistocene hominids. I. Mandibular molars: crown area measurements and morphological traits. J. Anat. 136 (1), 197-219.

Wood, B.A., Uytterschaut, H., 1987. Analysis of the dental morphology of

Plio-Pleistocene hominins. III. Mandibular premolar crowns. J. Anat.

154, 121-156.

Y'Edinak, G., 1978. Culture, diet, and dental reduction in Mesolithic forager-

fishers of Yugoslavia. Curr. Anthropol. 19, 616-618.

Zelditch, M.L., Sheets, H.D., Fink, W.L., 2003. The ontogenetic dynamics of

shape disparity. Paleobiology 29, 139-156.

Zelditch, M.L., Swiderski, D.L., Sheets, H.D., Fink, W.L., 2004. Geometric

Morphometrics for Biologists: A Primer. Elsevier Academic Press, San Diego. 